\title{
Association between Deep White Matter Hyperintensities and Right-to-left Shunt in Migraine (CAMBRAIN): A Cross-sectional Multicenter Study
}

Yingqi Xing ( $\nabla$ xingyq2009@sina.com )

Xuanwu Hospital, Capital Medical University https://orcid.org/0000-0002-7403-5789

\section{YiShui Zhang}

Jilin University First Hospital

HongLing Zhao

Dalian Municipal Central Hospital Affiliated of Dalian Medical University

\section{SiBo Wang}

Jilin University First Hospital

\section{YingHua Cui}

The Hospital of Yanbian University

\section{YaLang Fang}

The First Hospital of Shanxi Medical University

\section{Rong Guo}

People's Hospital of Liaoning Province

\section{YaJie Li}

the Centre Hospital of Jilin City

\section{Qi Wan}

The First Affiliated Hospital of Nanjing Medical University

\section{Kaili Zhang}

Shanxi Bethune Hospital Affiliated to Shanxi Medical University

\section{Yan Cui}

The Affiliated Hospital of Inner Mongolia Medical University

\section{HuiXing Wei}

The First Affiliated Hospital of Fujian Medical University

\section{Pan Lin}

The Second Hospital of Longyan City

\section{Yong You}

The Second Hospital of Hainan Medical University

\section{Hong Huang}

The First People's Hospital of Zunyi

\section{Yan Su}


The Third Affiliated Hospital of Gansu University of Chieses Medicine

\section{Research article}

Keywords: Migraine, Right-to-left shunt, White matter hyperintensity, Transcranial Doppler

Posted Date: January 19th, 2021

DOl: https://doi.org/10.21203/rs.3.rs-147105/v1

License: (c) (i) This work is licensed under a Creative Commons Attribution 4.0 International License. Read Full License 


\section{Abstract}

\section{Background}

Deep white matter hyperintensities (DWMHs), often identified by hyperintense lesions on T2-weighted magnetic resonance imaging (MRI), were discovered to have a higher prevalence in migraine patients. A right-to-left shunt (RLS), which is also prevalent in migraineurs, could potentially contribute to the formation of DWMHs by induction of controversial embolism and endothelial dysfunction. In this crosssectional study, we aim to evaluate the association between RLS and the prevalence of DWMHs in patients with migraine.

Methods

In this study, we consecutively enrolled patients with migraine aged between 18 and 50 years from the 14 headache clinics of participating hospitals. DWMHs were rated using Scheltens scale on digital MRI images obtained from 1.5T scanners, and RLS was detected via contrast-enhanced transcranial Doppler. Analyses on DWMH prevalence and loads by RLS grading or subtype were performed. A logistic regression analysis on DWMH prevalence was also performed.

Results

In all, 237 migraine patients (age: $39.3 \pm 11.7,78.1 \%$ women, $13 \%$ migraine with aura) were enrolled. RLS was detected in $48.5 \%$ of the subjects and DWMHs were identified in $138(58.2 \%)$ patients. Prevalence of DWMHs did not differ significantly between $\mathrm{RLS}^{+}(57.4 \%)$ and $\mathrm{RLS}^{-}$patients $(59.0 \%, \mathrm{p}=0.74)$. No statistical difference in DWMH loads was found between different RLS grades or subtypes. Instead of RLS grades $(p=0.75)$, age (OR 1.067; 95\% Cl 1.034-1.101; $p<0.001)$ and aura (OR 4.063; 95\%Cl 1.492$11.061 ; p=0.006$ ) were statistically significant independent risk factors for increased DWMH prevalence in migraine patients.

\section{Conclusions}

Our findings do not support an association between RLS and DWMHs in migraine patients, regardless of RLS grades or subtypes.

Clinical Trial Registration: NCT 03418766; Date of registration: February 1, 2018

\section{Introduction}

Migraine is a common headache disorder and causes a great burden of disability (1), yet the pathology of the disease still remains unclear. Recent literature reveals that migraine is a neurological disorder, and multiple brain areas including cortex, thalamus, hypothalamus, and brain stem are involved in different phases of a migraine attack (2). Also, structural changes including deep white matter hyperintensities (DWMHs) and subclinical brain infarctions were found associated with migraine $(3,4)$. A higher 
prevalence of subclinical focal DWMHs has been reported in migraine patients $(5,6)$. Moreover, this heavier burden of DWMHs has been associated with female and elderly patients $(3,7)$. Nevertheless, the higher prevalence of DWMHs in the migraine population cannot be fully explained by traditional vascular risk factors. It has been proposed that microvascular ischemia are caused by recurrent hemodynamic changes (8).

A right-to-left shunt (RLS) is a situation where an abnormal pathway between pulmonary and systemic circulations exists. Contrast-enhanced transcranial Doppler (c-TCD), with its high sensitivity and specificity for detecting both intracardiac and extracardiac shunts, is widely used in the detection of RLS. Patent foramen ovale, present in $20-25 \%$ of the general population and $40-60 \%$ of migraine patients (912), is usually accountable for intracardiac RLS. A higher reported prevalence of RLS in patients with migraine has led to the hypotheses that RLS could trigger migraine attacks in some patients and also contribute to the formation of DWMHs, by the induction of controversial embolism and endothelial dysfunction $(7,9,10)$. Observations on the association between RLS and WMHs have been made, with highly varying conclusions. In this study, we evaluated the association between the presence, grades, and subtypes of RLS and the prevalence of DWMHs in patients with migraine.

\section{Methods}

\section{Study design}

This is a cross-sectional, observational study, which was performed in 14 hospitals from July 2018 to March 2020 (see Additional file 1).

\section{Participants}

In this study, we consecutively enrolled patients aged between 18 and 55 years, and the recruitment procedure is shown in a flowchart (Fig. 1). The inclusion criteria for our study were 1) diagnosis of migraine by a neurologist from a headache clinic according to the International Classification of Headache Disorders III (13); 2) ability to provide informed consent to participate in the study; and 3) completion of the required tests, including brain MRI, carotid artery ultrasonography, transcranial Doppler (TCD), and C-TCD examinations. The exclusion criteria of our study were 1) a neurological disorder involving inpatient history; 2 ) severe artery stenosis detected by TCD or carotid artery ultrasonography; and 3 ) conditions that result in an inability to undergo a standard c-TCD examination, including an insufficient temporal window, inadequate cubital venous access, and inability to perform the Valsalva maneuver (VM) because of a severe heart or lung disease.

\section{Demographics, migraine features, and other clinical information measurement}

A standard questionnaire on demographic information and migraine features was conducted under guidance of a neurologist. Clinical histories including hypertension, hyperlipidemia, heart disease, 
diabetes, and stroke or transient ischemic attack (TIA) were collected based on self-reported history or doctor diagnoses. The Hamilton Depression and Anxiety Rating scales and the Montreal Cognitive Assessment scale were used to assess mood and anxiety symptoms and cognitive status, respectively, during the interview.

\section{TCD protocol}

In order to achieve a standard procedure in TCD examination, experienced TCD investigators from participating centers underwent a training program for 3 months at the First Hospital of Jilin University before the patient enrollment started. The investigators were blind to the migraine status of patients. TCD examinations followed our standard procedure using a TCD detector (EMS-9A; Delica, China) at all participating centers, and the same machine was used to conduct C-TCD to detect RLS. The procedure of C-TCD has been detailed in our previous work (10). Briefly, a hand-held $2 \mathrm{MHz}$ probe was used to monitor the left middle cerebral artery with the patient in a supine position. A mixture of $9 \mathrm{~mL}$ of normal saline solution, $1 \mathrm{~mL}$ of air, and a drop of the patient's own blood was used as our medium. The procedure was performed first with normal breathing, and then two more times with a $10 \mathrm{~s} \mathrm{VM}$, with a 5 -min interval between each procedure. RLS was diagnosed when at least one microbubble (MB) was detected in the TCD spectrum. Permanent RLS was characterized by an RLS detected at rest, whereas a provoked RLS was characterized by RLS only detected after a VM. Several category systems for C-TCD grading have been used $(11,14)$. Based on the standards reported by Wessler et al., Spencer et al., and our previous research $(10,15,16)$, we assessed RLS with a five-level category system: grade $0=$ negative; grade $\mathrm{I}=1 \leq$ MBs $\leq 10$; grade $\mathrm{II}=10<\mathrm{MBs} \leq 25$; grade $\mathrm{III}>25 \mathrm{MBs}$ and no curtain; and grade $\mathrm{IV}=$ curtain (when a single bubble cannot be identified). In this study, the c-TCD results are presented as RLS ${ }^{-}$(grade 0 ), smallto-moderate shunts (grade I and grade II), and large shunts (grade III to grade IV) (Fig. 2).

\section{MRI protocol and DWMHs rating}

Whole-brain MRI images (thickness $=5 \mathrm{~mm}$ ) on 1.5T scanners were obtained; on the axial plane, sequences included were pulse sequences of diffusion weighted sequences (DWI and ADC), FLAIR T2weighted sequences, and spin echo T1-weighted sequences. On both axial and sagittal planes, a spin echo T2-weighted sequence was obtained. Two neuro-radiologists blinded to any clinical information evaluated the digital images for prevalence and grades of DWMHs. DWMHs were defined as hyperintense lesions located in the supratentorial deep white matter, present on both standard and FLAIR T2-weighted sequences, with normal or slightly low signal intensities on T1-weighted sequences (Fig. 3).

Periventricular white matter hyperintensities, defined as WMH within $10 \mathrm{~mm}$ of the ventricular system, were excluded from this study. Virchow-Robin spaces were excluded as lesions with T2 hyperintensity that were not hyperintense on FLAIR T2 sequence. DWMHs were rated in each cerebral lobe following the Scheltens scale, and were scored as follows: 0) no lesions; 1 ) $<3 \mathrm{~mm}$ and $\mathrm{n}<6$; 2 ) $<3 \mathrm{~mm}$ and $\mathrm{n} \geq 6$; 3 ) 4-10 mm and $n<6$; 4) 4-10 mm and $n \geq 6$;5) $\geq 11 \mathrm{~mm}$ and $n \geq 1$; and 6) confluent lesions (17). Lesions found at basal ganglia and infratentorial spaces were not included in this study. Final DWMH load scoring values from two raters were averaged. 


\section{Statistical analysis}

We used Spearman's $\chi^{2}$, 2-tailed Fisher's exact, unpaired t, and Mann-Whitney $U$ tests to evaluate differences in the distributions and means of characteristics between study groups when appropriate. Using logistic regression analysis, we examined the odds ratio (OR) and 95\% confidence intervals (Cl) for DWMH presence based on RLS grading ( $\mathrm{RLS}^{-}$/small-to-moderate shunt/large shunt), sex, age, BMI, disease duration, attack frequency, migraine subtypes (migraine with aura [MwA] and migraine without aura [MwoA]), family history, hypertension, hyperlipidemia, and cognitive impairment. All analyses were conducted with IBM SPSS 24.0 (Chicago, IL, USA), and significance level was set at $p<0.05$.

\section{Results}

\section{Clinical characters of participants}

In all, 237 participants were included in the statistical analysis, with a mean age of $39.3 \pm 11.7$ years, of which 78.1\% were women. Among all 237 participants, 107 (45.1\%) were diagnosed for the first time with migraine, 32 (13.5\%) were diagnosed with MwA, 22 (9.3\%) with chronic migraine, and 7 (3.0\%) with medication-overuse migraine.

Eight patients had missing data, 1 on the use of oral contraceptives, 3 on caffeine intake, and 4 on exercise habits, all of whom were diagnosed with MwoA. These cases were removed in the analyses of specific variables.

\section{Association between RLS and DWMH}

Overall, 115 (48.5\%) patients had RLS, 52 (45.2\%) of whom had large shunts. RLS was detected in $48.3 \%$ of MwoA patients and $50 \%$ of MwA patients, with no statistically significant differences between migraine subtypes.

DWMHs were identified in 138 patients (58.2\%). Between the $\mathrm{RLS}^{+}$and $\mathrm{RLS}^{-}$groups, the prevalence rate of DWMHs did not differ significantly $\left(\mathrm{RLS}^{+} 57.4 \%\right.$ vs $\left.^{\mathrm{RLS}}{ }^{-} 59.0 \%, \mathrm{p}=0.74\right)$. We also found no statistically significant association between DWMHs and RLS subtypes or grades (Table 1). 
Table 1

Prevalence of DWMH on different subtypes and grades of RLS

\begin{tabular}{|llll|}
\hline & $\begin{array}{l}\mathrm{DWMH}^{-} \\
\mathbf{n}(\%)\end{array}$ & $\begin{array}{l}\mathrm{DWMH}^{+} \\
\mathbf{n}(\%)\end{array}$ & p value \\
\hline $\mathrm{RLS}^{-}$ & $50(41.0)$ & $72(59.0)$ & \\
\hline Small-to-moderate shunt & $46(41.3)$ & $37(58.7)$ & 0.97 \\
\hline Large shunt & $23(44.2)$ & $29(55.8)$ & 0.69 \\
\hline Permanent RLS & $23(43.4)$ & $30(56.6)$ & 0.79 \\
\hline Provoked RLS & $26(41.9)$ & $36(58.1)$ & 0.98 \\
\hline $\begin{array}{l}\text { DWMH: deep white matter hyperintensity; RLS: right-to-left shunt. } \mathrm{p}<0.05 \text { indicates statistical } \\
\text { significance. }\end{array}$ & & \\
\hline
\end{tabular}

As assessed using Scheltens scale, DWMH loads in our study ranged from 0 to 17 , with a median of 3 , and the 25th and 75th percentiles were 2 and 5, respectively. We divided patients with DWMHs into two groups: high DWMH loads ( $\geq 4$ on the scale) and low DWMH loads (1-3 on the scale). The proportion of participants with low and high DWMH loads was $34.6 \%$ and $23.6 \%$ of the sample, respectively. No statistically significant differences in DWMH loads were found with regard to RLS grades $(p=0.88)$ or subtypes $(p=0.54)$. Further, $23(36.5 \%)$ patients with small-to-moderate shunts had low DWMH loads, and $14(22.2 \%)$ had high DWMH loads. In contrast, 19 (36.5\%) patients with large shunts had low DWMH loads and10 (19.2\%) had high DWMH loads (Fig. 4a). Twenty-two (41.5\%) and 8 (15.1\%) patients with permanent RLS had low and high DWMH loads, respectively. Among patients with provoked RLS, 20 (32.3\%) and 16 (25.8\%) had low and high DWMH loads, respectively (Fig. 4b).

\section{Clinical characteristics in $\mathrm{DWMH}^{+}$and $\mathrm{DWMH}^{-}$patients}

First, we analyzed the differences in all demographic, migraine-related, and other clinical characteristics between the $\mathrm{DWMH}^{+}$and $\mathrm{DWMH}^{-}$groups. Significant differences in $\mathrm{DWMH}$ prevalence with regard to age, BMI, disease duration, and family history were found in single variable analyses (Table 2). Diabetes and coronary heart disease could not be analyzed because of the small sample size (one patient in the $\mathrm{DWMH}^{-}$group had coronary heart disease; 7 patients had diabetes: 4 in the $\mathrm{DWMH}^{+}$group and 3 in the $\mathrm{DWMH}^{-}$group). None of the participants reported a history of stroke or TIA. 
Table 2

Characteristics of patients with and without DWM

$\mathrm{DWMH}^{+}$

$\mathrm{DWMH}^{-}$

p value

$n=138 \quad n=99$

\section{Demographics}

Age, years

$44(34-50)$

$34(27-45)$

$<0.001$

Sex, female

$110(79.7)$

75 (75.8)

0.47

BMl, $\mathrm{kg} / \mathrm{m}^{2}$

$23.0(20.8-24.8)$

$22.0(20.2-24.2) \quad 0.046$

Low education

$9(6.5)$

$8(8.1)$

0.64

Migraine features

Disease duration, years

$10.0(5.0-18.0)$

$7.5(3.5-15.0)$

0.030

Attack frequency (per month in last 3 months)

$2(1-3)$

$1(0-4)$

0.33

HIT-6

$56(50-61)$

$57(52-63)$

0.23

MIDAS

$5(1-10)$

$5(0-10)$

0.90

Migraine with aura

$23(16.7)$

$9(9.1)$

0.092

Medication

65 (53.3)

$73(63.5)$

0.11

Family history

$20(14.5)$

$33(33.3)$

0.001

Vascular risk factors and other clinical features

Current smoker

18 (13.0)

$8(8.1)$

0.23

Hypertension

$8(5.8)$

$2(2.0)$

0.20

Hyperlipidemia

$12(8.7)$

3 (3.0)

0.077

Oral contraceptive use

5 (4.6)

3 (2.7)

0.70

Alcohol drinking

9 (6.5)

2 (2.0)

0.13

Caffeine intake

5 (3.6)

8 (8.2)

0.13

Exercise

31 (23.1)

15 (15.2)

0.13

DWMH: deep white matter hyperintensity. BMI: body mass index. Low education: less than primary school education or education years under 6. HIT-6: Headache impact test. MIDAS: the migraine disability assessment test. Alcohol drinking: alcohol consumption over $25 \mathrm{~g}$ for male or $15 \mathrm{~g}$ for female per week. Caffeine intake: drinking tea or coffee at less than one cup per day. Exercise: $\geq 30$ minutes of exercise per day for at least 3 days/week. Depression and Anxiety: assessed by Hamilton Depression Rating Scale (17 items) and Hamilton Rating Scale for Anxiety. Cognitive impairment: assessed by Montreal Cognitive Assessment scale. 


\begin{tabular}{|c|c|c|c|}
\hline & $\mathrm{DWMH}^{+}$ & $\mathrm{DWMH}^{-}$ & \multirow[t]{2}{*}{$\mathrm{p}$ value } \\
\hline & $n=138$ & $n=99$ & \\
\hline Depression & $13(9.4)$ & $13(13.1)$ & 0.37 \\
\hline Anxiety & $24(17.4)$ & $24(24.2)$ & 0.20 \\
\hline Cognitive impairment & $27(19.6)$ & $26(26.3)$ & 0.22 \\
\hline \multicolumn{4}{|c|}{$\begin{array}{l}\text { DWMH: deep white matter hyperintensity. BMI: body mass index. Low education: less than primary } \\
\text { school education or education years under } 6 \text {. HIT- } 6 \text { : Headache impact test. MIDAS: the migraine } \\
\text { disability assessment test. Alcohol drinking: alcohol consumption over } 25 \mathrm{~g} \text { for male or } 15 \mathrm{~g} \text { for } \\
\text { female per week. Caffeine intake: drinking tea or coffee at less than one cup per day. Exercise: } \geq 30 \\
\text { minutes of exercise per day for at least } 3 \text { days/week. Depression and Anxiety: assessed by Hamilton } \\
\text { Depression Rating Scale ( } 17 \text { items) and Hamilton Rating Scale for Anxiety. Cognitive impairment: } \\
\text { assessed by Montreal Cognitive Assessment scale. }\end{array}$} \\
\hline
\end{tabular}

Values in this table are expressed as $n(\%)$; or median (25th -75 th percentiles). $p<0.05$ indicates statistical significance.

Next, logistic regression analysis on DWMH prevalence was performed. Variables considered to have either a statistical or clinical association with DWMHs were included. For the included variables, a correlation between disease duration and age was found $(p<0.001)$, and family history was found to be associated with younger age [37 $(28-45)$ years vs $42(31-48)$ years; $p=0.034]$ and longer disease duration [10 (6-20) years vs $8(3.5-15)$ years; $p=0.006]$.

After controlling for other variables, age remained as an independent risk factor for the presence of DWMHs. There was also a positive association between a diagnosis of MwA and the presence of DWMHs. No statistically significant association between RLS grading and DWMHs was found, and family history showed a negative association with DWMHs, statistically (Table 3). 
Table 3

Multivariable logistic regression analyses for the presence of DWMHs

\begin{tabular}{|llll|}
\hline & p value & OR & $95 \%$ Cl \\
\hline RLS grading & 0.75 & & \\
\hline Small-to-moderate shunt & 0.48 & 1.293 & $0.638-2.620$ \\
\hline Large shunt & 0.62 & 1.213 & $0.572-2.571$ \\
\hline Age (per year) & $<0.001$ & 1.067 & $1.034-1.101$ \\
\hline BMI & 0.106 & 1.094 & $0.981-1.219$ \\
\hline Female & 0.87 & 1.065 & $0.500-2.269$ \\
\hline Disease duration & 0.40 & 1.017 & $0.978-1.057$ \\
\hline Migraine with aura & 0.006 & 4.063 & $1.492-11.061$ \\
\hline Attack frequency & 0.29 & 0.969 & $0.916-1.026$ \\
\hline Family history & 0.020 & 0.416 & $0.199-0.869$ \\
\hline Hypertension & 0.80 & 1.249 & $0.220-7.096$ \\
\hline Hyperlipidemia & 0.46 & 1.737 & $0.399-7.568$ \\
\hline Cognitive impairment & 0.062 & 0.483 & $0.224-1.038$ \\
\hline $\begin{array}{l}\text { DWMHs: deep white matter hyperintensities; } \text { RLS: right-to-left shunt; BMl: body mass index. } \mathrm{p}<0.05 \\
\text { indicates statistical significance. }\end{array}$ & & \\
\hline
\end{tabular}

\section{Discussion}

Through this observational study, we aimed to clarify if there is an association between DWMHs and RLS in migraine patients, especially in those with large or permanent shunts. The prevalence rate of DWMHs in our study was $58.2 \%$, which is slightly higher than that reported in previous studies (7), despite the younger age (39.3 \pm 11.7 years) of our participants. The overall prevalence of RLS in our study $(48.5 \%)$ was similar to that of our previous work (46.1\%) (10), but lower compared to other studies $(9,18)$. Also, a higher prevalence rate of RLS in patients diagnosed with MwA, as reported in the literature $(10,19)$, was not found in our study. This was probably because of the limited sample size of patients with MwA. Based on our findings, DWMHs in migraine patients have no association with RLS, regardless of the subtypes or grades. Neither patients with permanent RLS nor those with large shunts have an increased risk of presenting a higher prevalence of DWMHs. After controlling for other variables, age and aura seem to be independent risk factors for an increased prevalence of DWMHs.

\section{RLS and DWMHs in migraine}


WMHs have always been considered as a phenomenon of small vessel disease (20), and the most consistent risk factors for WMHs are age and hypertension (21). Current literature suggests that migraine is a risk factor for WMHs $(5,22)$, raising questions regarding whether migraine patients are more vulnerable to the development of WMHs and how these may affect them.

The association between RLS and WMHs in migraine patients has long been debated. The majority of published papers have reported no association between $\operatorname{RLS}$ and WMHs $(7,9,23)$; however, some research has shown a positive association $(24,25)$. There is evidence that migraine patients have a higher prevalence of $\operatorname{RLS}(9,10)$, and it is believed that paradoxical embolism caused by RLS is related to cryptogenic stroke and silent brain infarcts in migraine (26). Therefore, some authors suggest that RLS is involved in WMHs through a similar mechanism (27).

However, as no widely accepted theory on the pathology of WMHs has been proposed, it is possible that periventricular WMHs (PVWMHs) and DWMHs have differences in pathology, according to neuroimaging studies (28). High burdens of PVWMHs are more often associated with clinically isolated syndromes and cognitive impairment diseases $(28,29)$, whereas DWMHs have a higher prevalence among migraine patients. In the CAMERA series studies, only DWMHs in women were found to have a different prevalence between migraineurs and controls $(3,5)$. Furthermore, DWMH lesions that seem to progress in migraine patients as pre-existing microstructural white matter changes have been observed years before the emergence of visible focal WMHs on conventional MRI (30). Furthermore, a study on migraine and tension-type headache patients suggested that small DWMHs are associated with RLS in young migraineurs (25). Therefore, we may assume that if paradoxical embolisms caused by RLS play a part in WMHs in migraine patients, it is most likely associated with DWMHs, rather than PVWMHs. In addition, similar to the case in cryptogenic stroke, patients with permanent RLS and large shunts should be at a higher risk $(31,32)$.

In this study, C-TCD instead of heart sonography is used to detect RLS due to its higher sensitivity (33). Although c-TCD cannot distinguish the different levels of RLS, both intracardiac and extracardiac shunts can cause paradoxical embolism. Moreover, compared to heart sonography, c-TCD has a better detection rate on extracardiac shunts (34). A different system for rating RLS grades was used in this study instead of the one that most former studies on this topic have used, mainly dividing RLS into three grades: negative, less than $10 \mathrm{MBs}$, and more than $10 \mathrm{MBs}$. This decision was based on our former research, which has shown that the prevalence rate of large shunts (defined as $>25 \mathrm{MBs}$ or curtain on TCD spectrum) in migraineurs is significantly higher than that in healthy controls (10). Thus, when it comes to a possible paradoxical embolism caused by RLS, we believe that large shunts under our category system should more likely be deemed a probable cause. However, results from our study add further evidence to the lack of association between RLS and DWMHs, even in patients with large or permanent shunts. Therefore, it is not likely that RLS contributes to the high prevalence of DWMHs in the migraine population through paradoxical embolism.

\section{Age, sex, and cognitive impairment}

Page $11 / 18$ 
In concordance with previous findings $(7,35)$, a positive association between age and DWMH prevalence was found in our study (OR: 1.067, 95\% Cl: 1.035-1.101, $p<0.001$; per year).

Whether this higher burden of DWMHs is a consequence of normal aging or migraine as a risk factor remains unclear. There is consistent evidence of age-related accumulation of both PVWMH and DWMH burden $(21,36)$, suggesting that WMHs might be part of the normal aging pattern in brain tissue. However, an imaging pattern study on brain aging in the general population has shown that WMHs contribute to beyond-normal brain aging imaging findings (36). DWMH progressions in migraine patients have been reported in some longitudinal studies, suggesting that female migraineurs or patients with recurrent headache attacks may develop heavier DWMH burdens $(3,4)$. Therefore, it is possible that this association between age and DWMHs in migraine patients is an outcome of both aging and the impacts of migraine-related factors over the years.

The difference between sexes, as reported in several studies $(6,30)$, was not detected in our study. A possible reason is the different proportion of women on oral contraceptives in different study populations. In our study, only 8 out of the 185 women reported a history of oral contraceptive use, compared with $25 \%$ of women who had a history of over 15 years of oral contraceptive use in the CAMERA study (30). Considering that the use of oral contraceptives can be a risk factor for vascular incidents, the low rate of oral contraceptive usage in our study group could account for this difference.

We did not find any association between DWMH prevalence and cognitive impairment, which may be explained by the mild load of DWMHs in migraine, in line with a previous study (3). Furthermore, a recent study on neuroimaging suggests that instead of DWMHs, PVWMHs are associated with cognitive impairment (28).

\section{Migraine features}

With regard to migraine features, we found a positive association between DWMHs and aura, which is consistent with a previous finding (37). Cortical hyper-excitability is thought to lower the threshold for cortical spreading depression, which could be responsible for aura in migraine. This cortical spreading depression might be caused by ischemic events, which possibly links the association of aura with DWMHs (38).

Disease duration and attack frequency, as reported in the literature $(9,24)$, were found to have no association with DWMHs in our study. Contrary to what we expected, we found a negative association with family history. However, it must be mentioned that patients with a self-reported positive family history in our study were associated with younger age and longer disease duration. Therefore, it would be too early to draw a conclusion based on our current data.

\section{Strengths and limitations}

This is a cross-sectional multicenter study with 14 participating hospitals, which to some extent makes our data representative of Chinese migraine patients from headache clinics. To the best of our 
knowledge, few reports on this issue have focused on patients with large shunts. Therefore, our study adds new evidence clarifying that RLS was found to have no association with DWMHs in migraine even in patients with large shunts ( $>25 \mathrm{MBs}$ or curtain on spectrum).

Our study presents a series of limitations. First, this study is lacking in healthy controls due to the difficulty in obtaining MRI images from healthy populations. Since we focused on the association between RLS and DWMH in migraine patients, our conclusion still has some reference value in clinical practice. Second, because of our limited sample size, only $13.5 \%$ of the patients were diagnosed with MwA. In addition, as $45 \%$ of our participants were diagnosed with migraine for the first time, participants in our study have suffered from severe migraine attacks less often. Therefore, conclusions from our study may not be applicable to severe migraine patients. For future research, work on DWMH progression in different subtypes of migraine and in patients and controls who have undergone patent foramen ovale closure surgery should be conducted.

\section{Conclusions}

Results from our study indicate that when DWMHs are discovered in migraine patients, RLS may not be the etiological cause, even in patients with large shunts. Our study suggests that only age and aura are associated with DWMH presence.

\section{Declarations}

\section{Ethics approval and Consent to participate}

Ethical approvals for this study were obtained from each of the ethics boards of the participating hospitals. This study was registered on clinicaltrial.gov (Clinical trial No.: NCT 03418766; Date of registration: February 1,2018$)$. Written informed consents were provided by all patients.

\section{Consent for publication}

This manuscript does not contain any data that could reveal a participating individual's personal information.

\section{Availability of data and materials}

The datasets used and analyzed during the current study are available from the corresponding author on reasonable request.

\section{Competing interests}

The authors declare that they have no competing interests.

\section{Funding}


This work was supported by the Natural Science Foundation of China (NSFC, Grant No. 81971620).

\section{Authors' contributions}

ZYS analyzed and interpreted the patient data, and was a major contributor in writing the manuscript. XY and WSB contributed to the original idea of this work, and provided guidance in data interpretation and manuscript writing. All other listed authors were contributors in patients' enrollment and data acquirement. All authors read and approved the final manuscript.

\section{Acknowledgments:}

We would like to thank all staff from the headache clinics of the 14 hospitals for their help in enrollment and examination work.

\section{References}

1. Stovner LJ, Nichols E, Steiner TJ, Abd-Allah F, Abdelalim A, Al-Raddadi RM, et al. Global, regional, and national burden of migraine and tension-type headache, 1990-2016: a systematic analysis for the Global Burden of Disease Study 2016. The Lancet Neurology. 2018;17(11):954-76.

2. Charles A. The pathophysiology of migraine: implications for clinical management. The Lancet Neurology. 2018;17(2):174-82.

3. Palm-Meinders IH, Koppen H, Terwindt GM, Launer LJ, Konishi J, Moonen JME, et al. Structural brain changes in migraine. JAMA. 2012;308(18):1889-97.

4. Erdélyi-Bótor S, Aradi M, Kamson DO, Kovács N, Perlaki G, Orsi G, et al. Changes of migraine-related white matter hyperintensities after 3 years: a longitudinal MRI study. Headache. 2015;55(1):55-70.

5. Kruit MC, van Buchem MA, Hofman PAM, Bakkers JTN, Terwindt GM, Ferrari MD, et al. Migraine as a risk factor for subclinical brain lesions. JAMA. 2004;291(4):427-34.

6. Kruit MC, van Buchem MA, Launer LJ, Terwindt GM, Ferrari MD. Migraine is associated with an increased risk of deep white matter lesions, subclinical posterior circulation infarcts and brain iron accumulation: the population-based MRI CAMERA study. Cephalalgia. 2010;30(2):129-36.

7. Adami A, Rossato G, Cerini R, Thijs VN, Pozzi-Mucelli R, Anzola GP, et al. Right-to-left shunt does not increase white matter lesion load in migraine with aura patients. Neurology. 2008;71(2):101-7.

8. Aradi M, Schwarcz A, Perlaki G, Orsi G, Kovács N, Trauninger A, et al. Quantitative MRI Studies of Chronic Brain White Matter Hyperintensities in Migraine Patients. Headache: The Journal of Head and Face Pain. 2013;53(5):752-63.

9. Koppen H, Palm-Meinders IH, Mess WH, Keunen RW, Terwindt GM, Launer LJ, et al. Systemic right-toleft shunts, ischemic brain lesions, and persistent migraine activity. Neurology. 2016;86(18):1668-75.

10. Wang S-B, Liu K-D, Yang Y, Li Y-J, Hu M-Y, Lin P, et al. Prevalence and extent of right-to-left shunt on contrast-enhanced transcranial Doppler in Chinese patients with migraine in a multicentre casecontrol study. Cephalalgia. 2018;38(4):690-6. 
11. Hagen PT, Scholz DG, Edwards WD. Incidence and size of patent foramen ovale during the first 10 decades of life: an autopsy study of 965 normal hearts. Mayo Clin Proc. 1984;59(1):17-20.

12. Wammes-van der Heijden EA, Tijssen CC, Egberts ACG. Right-to-left shunt and migraine: the strength of the relationship. Cephalalgia. 2006;26(2):208-13.

13. Headache Classification Committee of the International Headache Society (IHS) The International Classification of Headache Disorders, 3rd edition. Cephalalgia. 2018;38(1).

14. Serena J, Marti-Fàbregas J, Santamarina E, Rodríguez JJ, Perez-Ayuso MJ, Masjuan J, et al. Recurrent stroke and massive right-to-left shunt: results from the prospective Spanish multicenter (CODICIA) study. Stroke. 2008;39(12):3131-6.

15. Xu WH, Xing YQ, Yan ZR, Jiang JD, Gao S. Cardiac right-to-left shunt subtypes in Chinese patients with cryptogenic strokes: a multicenter case-control study. European Journal of Neurology. 2014;21(3):525-8.

16. Spencer MP, Moehring MA, Jesurum J, Gray WA, Olsen JV, Reisman M. Power m-mode transcranial Doppler for diagnosis of patent foramen ovale and assessing transcatheter closure. J Neuroimaging. 2004;14(4):342-9.

17. Scheltens P, Barkhof F, Leys D, Pruvo JP, Nauta JJ, Vermersch P, et al. A semiquantative rating scale for the assessment of signal hyperintensities on magnetic resonance imaging. J Neurol Sci. 1993;114(1).

18. Jiang X-H, Wang S-B, Tian Q, Zhong C, Zhang G-L, Li Y-J, et al. Right-to-left shunt and subclinical ischemic brain lesions in Chinese migraineurs: a multicentre MRI study. BMC Neurol. 2018;18(1):18.

19. Wammes-van der Heijden EA, Tijssen CC, Egberts AC. Right-to-left shunt and migraine: the strength of the relationship. Cephalalgia. 2006;26(2):208-13.

20. Pantoni L. Cerebral small vessel disease: from pathogenesis and clinical characteristics to therapeutic challenges. The Lancet Neurology. 2010;9(7):689-701.

21. Gottesman RF, Coresh J, Catellier DJ, Sharrett AR, Rose KM, Coker LH, et al. Blood pressure and white-matter disease progression in a biethnic cohort: Atherosclerosis Risk in Communities (ARIC) study. Stroke. 2010;41(1):3-8.

22. Hamedani AG, Rose KM, Peterlin BL, Mosley TH, Coker LH, Jack CR, et al. Migraine and white matter hyperintensities: the ARIC MRI study. Neurology. 2013;81(15):1308-13.

23. Del Sette M, Dinia L, Bonzano L, Roccatagliata L, Finocchi C, Parodi RC, et al. White Matter Lesions in Migraine and Right-to-Left Shunt: A Conventional and Diffusion MRI Study. Cephalalgia. 2008;28(4):376-82.

24. Iwasaki A, Suzuki K, Takekawa H, Takashima R, Suzuki A, Suzuki S, et al. The relationship between right-to-left shunt and brain white matter lesions in Japanese patients with migraine: a single center study. J Headache Pain. 2017;18(1):3.

25. Park HK, Lee SY, Kim SE, Yun CH, Kim SH. Small deep white matter lesions are associated with rightto-left shunts in migraineurs. J Neurol. 2011;258(3):427-33. 
26. West BH, Noureddin N, Mamzhi Y, Low CG, Coluzzi AC, Shih EJ, et al. Frequency of Patent Foramen Ovale and Migraine in Patients With Cryptogenic Stroke. Stroke. 2018;49(5):1123-8.

27. Xie H, Bian Y, Jian Z, Huo K, Liu R, Zhu D, et al. Right-to-left shunt may be prone to affect the white matter integrity of posterior circulation in migraine without aura. Neurol Sci. 2018;39(1):119-25.

28. Griffanti L, Jenkinson M, Suri S, Zsoldos E, Mahmood A, Filippini N, et al. Classification and characterization of periventricular and deep white matter hyperintensities on MRI: A study in older adults. Neuroimage. 2018;170:174-81.

29. Lapucci C, Saitta L, Bommarito G, Sormani MP, Pardini M, Bonzano L, et al. How much do periventricular lesions assist in distinguishing migraine with aura from CIS? Neurology. 2019;92(15):e1739-e44.

30. Arkink EB, Palm-Meinders IH, Koppen H, Milles J, van Lew B, Launer LJ, et al. Microstructural white matter changes preceding white matter hyperintensities in migraine. Neurology. 2019;93(7):e688-e94.

31. Rigatelli G, Dell'Avvocata F, Cardaioli P, Giordan M, Braggion G, Aggio S, et al. Permanent right-to-left shunt is the key factor in managing patent foramen ovale. J Am Coll Cardiol. 2011;58(21):2257-61.

32. Anzola GP, Zavarize P, Morandi E, Rozzini L, Parrinello G. Transcranial Doppler and risk of recurrence in patients with stroke and patent foramen ovale. Eur J Neurol. 2003;10(2):129-35.

33. Katsanos AH, Psaltopoulou T, Sergentanis TN, Frogoudaki A, Vrettou AR, Ikonomidis I, et al. Transcranial Doppler versus transthoracic echocardiography for the detection of patent foramen ovale in patients with cryptogenic cerebral ischemia: A systematic review and diagnostic test accuracy meta-analysis. Ann Neurol. 2016;79(4):625-35.

34. de Havenon A, Moore A, Sultan-Qurraie A, Majersik JJ, Stoddard G, Tirschwell D. Ischemic stroke patients with active malignancy or extracardiac shunts are more likely to have a right-to-left shunt found by TCD than echocardiogram. Transl Stroke Res. 2015;6(5):361-4.

35. Komaromy H, He M, Perlaki G, Orsi G, Nagy SA, Bosnyak E, et al. Influence of hemispheric white matter lesions and migraine characteristics on cortical thickness and volume. J Headache Pain. 2019;20(1):4.

36. Habes M, Erus G, Toledo JB, Zhang T, Bryan N, Launer LJ, et al. White matter hyperintensities and imaging patterns of brain ageing in the general population. Brain. 2016;139(Pt 4):1164-79.

37. Kurth T, Mohamed S, Maillard P, Zhu Y-C, Chabriat H, Mazoyer B, et al. Headache, migraine, and structural brain lesions and function: population based Epidemiology of Vascular Ageing-MRI study. BMJ. 2011;342:c7357.

38. Eikermann-Haerter K. Spreading depolarization may link migraine and stroke. Headache. 2014;54(7):1146-57.

\section{Figures}




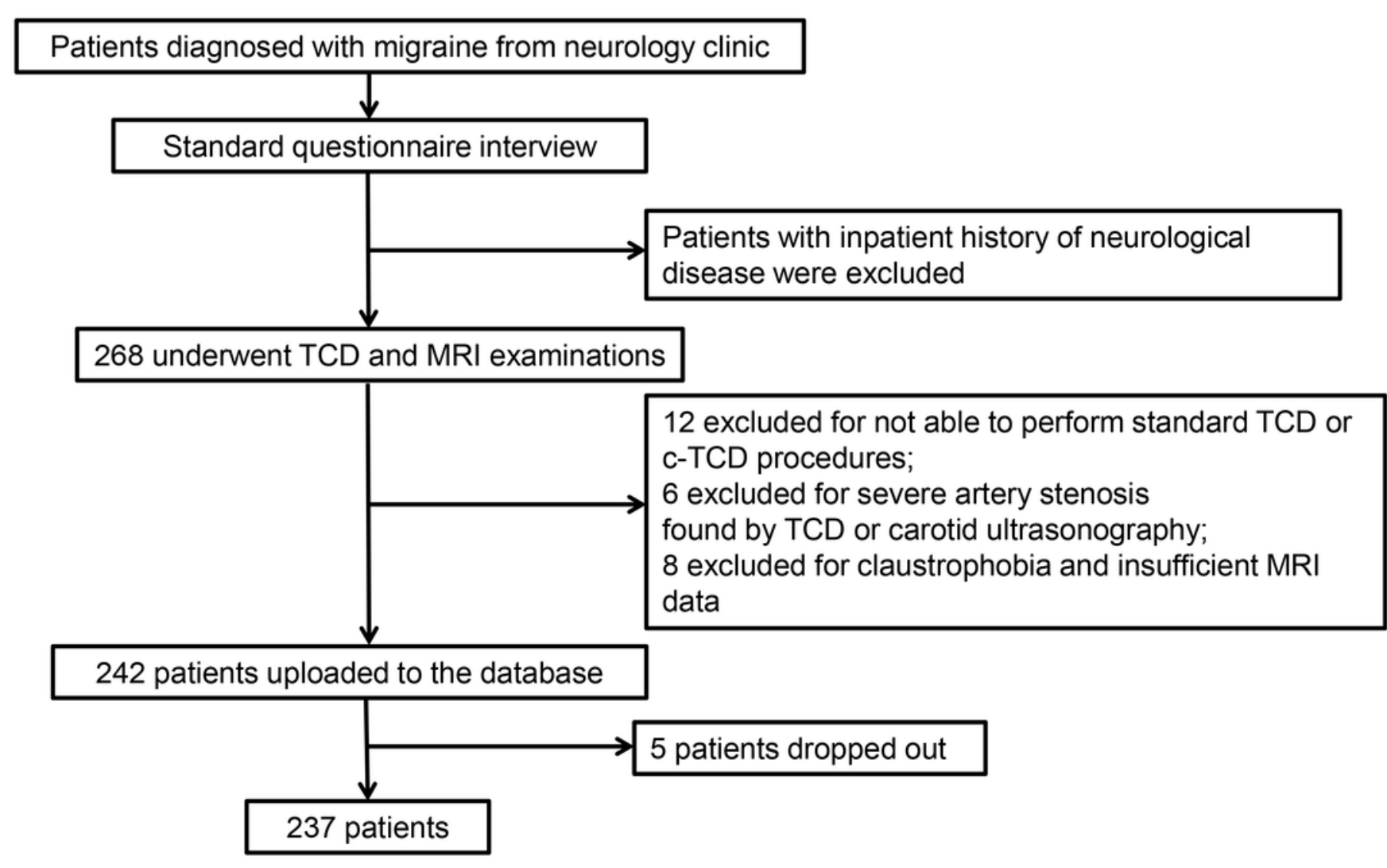

\section{Figure 1}

Flow chart of recruitment and participation in this study.

(a)

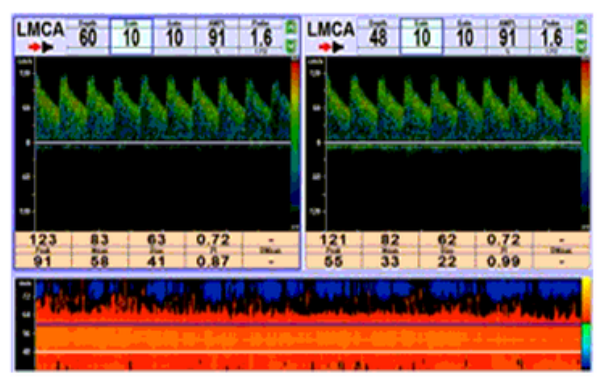

(d)

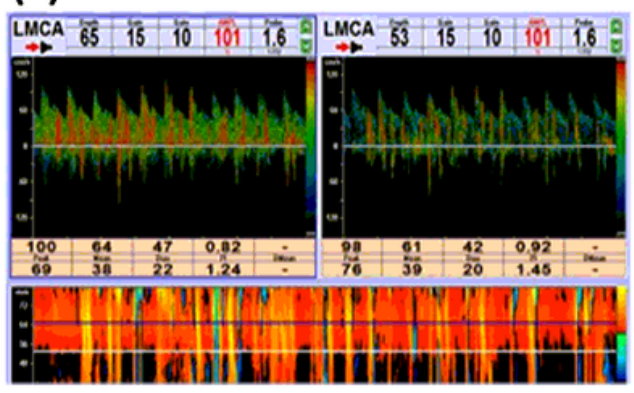

(b)

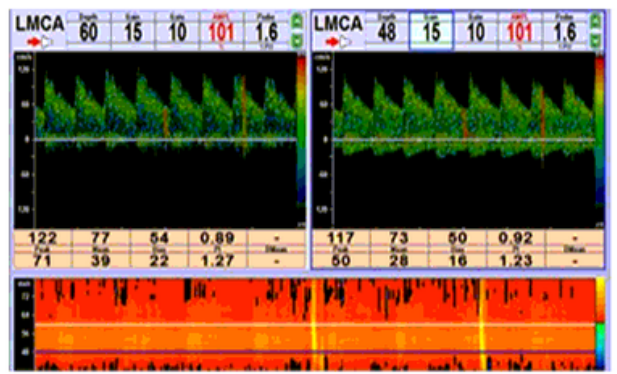

(e)

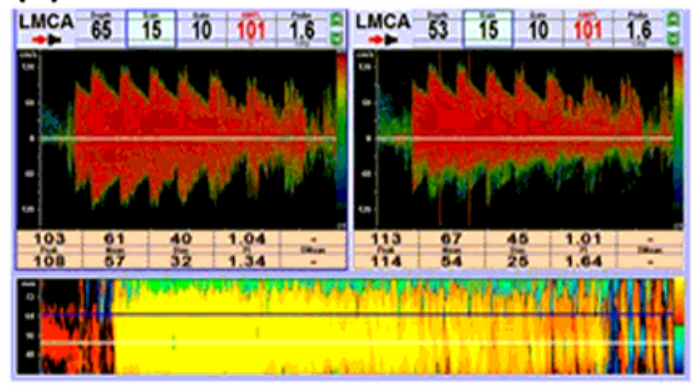

Figure 2

(c)

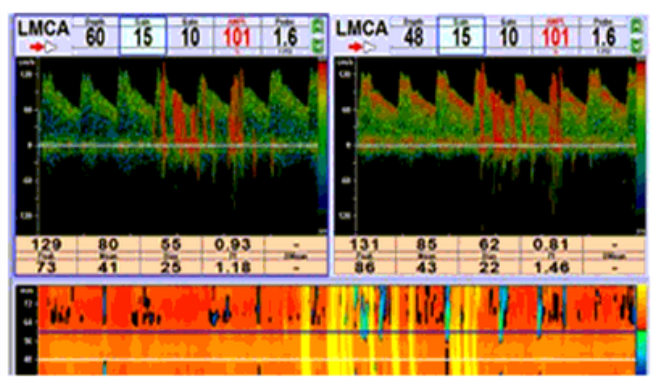


Category system of RLS under c-TCD. RLS-: grade $0=$ no MBs detected (a); small-to-moderate shunt: grade $\mathrm{I}=1 \leq \mathrm{MBs} \leq 10$, (b) and grade $\mathrm{II}=10<\mathrm{MBs} \leq 25$ (c); large shunt: grade $\mathrm{III}=>25 \mathrm{MBs}$ but no curtain (d) and grade IV = curtain (e). RLS, right-to-left shunt; c-TCD, contrast-enhanced transcranial Doppler; MB, microbubble

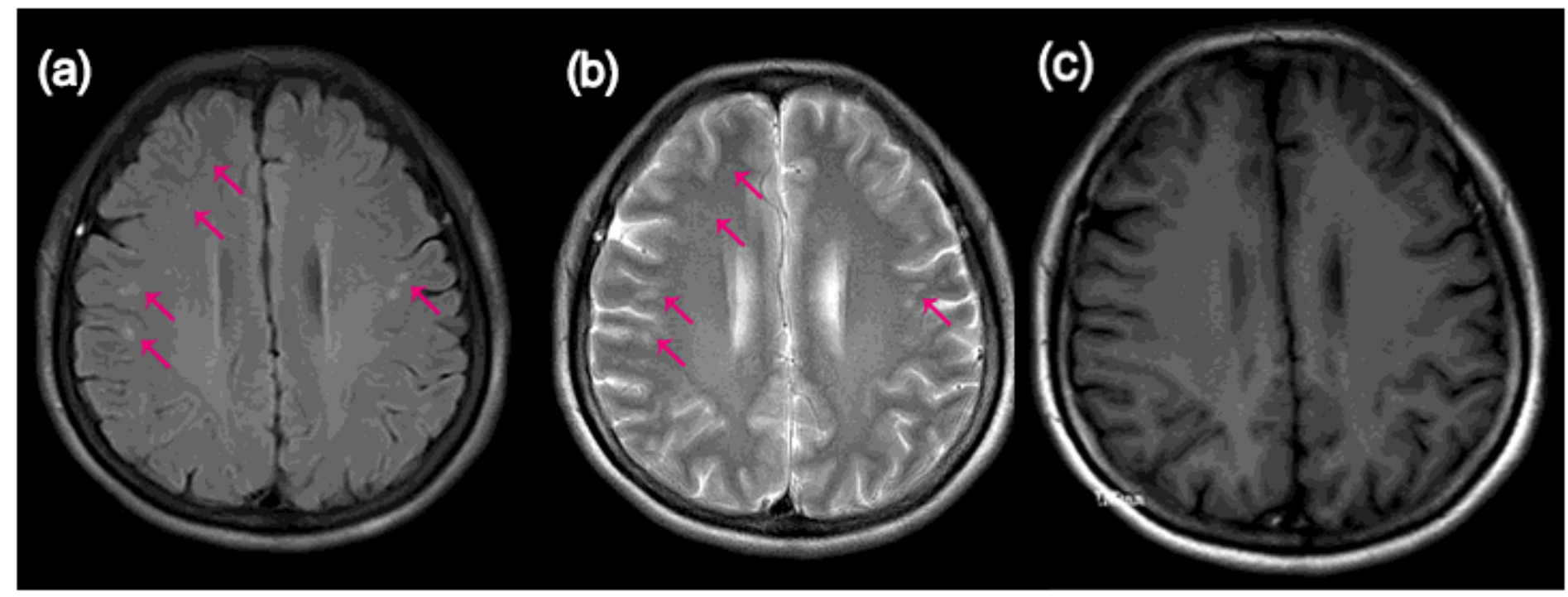

Figure 3

MRI images of deep white matter hyperintensity. Deep white matter hyperintensities were defined as hyperintense lesions located in the supratentorial deep white matter, present on both FLAIR T2-weighted sequences (a); and standard T2-weighted sequences (b); with normal or slightly low signal intensities on T1-weighted sequences (c).
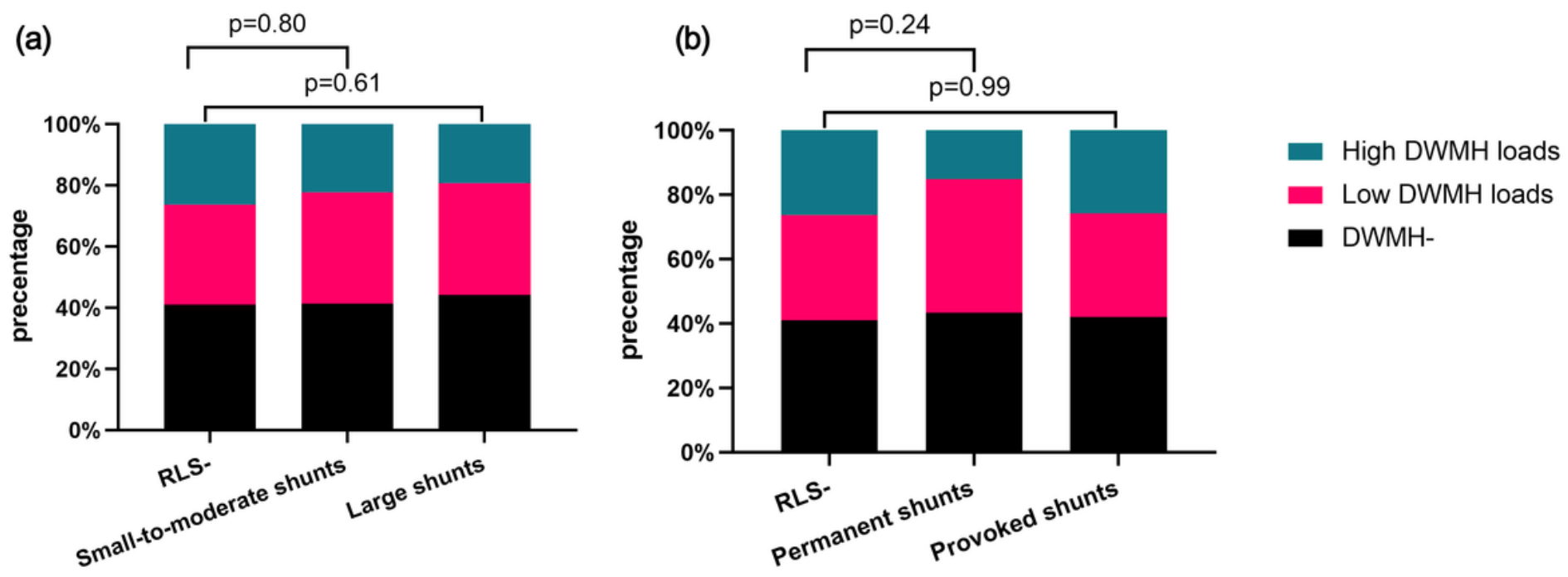

Figure 4

Distribution of deep white matter hyperintensity loads in different grades and subtypes of RLS patients. No statistically significant differences in DWMH loads were found with regard to RLS grades (a) or subtypes (b). DWMH: deep white matter hyperintensity; RLS, right-to-left shunt 